\title{
Latent Class Analysis of Functional Somatic Symptoms in a Population-Based Sample of Twins
}

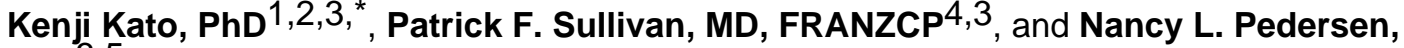 \\ $\mathrm{PhD}^{3,5}$ \\ ${ }^{1}$ School of Nursing and Rehabilitation, International University of Health and Welfare, Odawara, \\ Japan \\ ${ }^{2}$ Department of Health Promotion Science, Graduate School of Medicine, Osaka University, \\ Suita, Japan \\ ${ }^{3}$ Department of Medical Epidemiology and Biostatistics, The Karolinska Institute, Stockholm, \\ Sweden \\ ${ }^{4}$ Departments of Genetics, Psychiatry, \& Epidemiology, University of North Carolina at Chapel \\ Hill, USA \\ ${ }^{5}$ Department of Psychology, University of Southern California, USA
}

\begin{abstract}
Objective-This study aimed to investigate empirically how and in what way individuals with symptoms of functional somatic syndromes should be classified. We also aimed to look into genetic and environmental influences on the classification.

Method-A total of 28531 twins aged 41-64 underwent screening interviews via a computerassisted data collection system from 1998 to 2002. Nine functional somatic symptoms (abnormal tiredness, general muscular pain, recurrent abdominal discomfort, back pain, gastroesophageal reflux, recurrent headache, recurrent urinary problem, dizziness, breathlessness at rest) were assessed using structured questions in a blinded manner. Latent class analysis was applied to the data. Structural equation modeling was further performed in order to estimate the relative importance of genetic and environmental influences on class probability.
\end{abstract}

Results-Latent class analysis resulted in a 5-class solution. Individuals in the first class did not show any health problems. Those assigned to the second, third, and fourth classes tended to have abnormal tiredness, gastrointestinal problems, and pain-related symptoms, respectively. Individuals in the fifth class had multiple symptoms to a greater extent than the other classes. All the five classes showed modest genetic influences $(7-29 \%$ of the total variation) with gender differences except Class 3; however, the majority of influences on the class membership derived from unique environmental effects.

Conclusion-The findings suggested the necessity of re-defining the existing classification criteria for functional somatic syndromes in terms of single (uncomplicated) or multiple

(C) 2010 Elsevier Inc. All rights reserved.

*Correspond with Dr Kenji Kato at the Department of Health Promotion Science (c/o Prof Kazuo Hayakawa), Graduate School of Medicine, Osaka University, 1-7 Yamada-Oka, Suita 565-0871 Japan. kenji-kato@ umin.ac.jp.

This is a PDF file of an unedited manuscript that has been accepted for publication. As a service to our customers we are providing this early version of the manuscript. The manuscript will undergo copyediting, typesetting, and review of the resulting proof before it is published in its final citable form. Please note that during the production process errors may be discovered which could affect the content, and all legal disclaimers that apply to the journal pertain. 
(complicated) syndromes. Environmental influences are important for the aetiology of functional somatic syndromes.

\section{Keywords}

functional somatic syndromes; chronic fatigue syndrome; chronic widespread pain; irritable bowel syndrome; comorbidity; latent class analysis

\section{INTRODUCTION}

Functional somatic syndromes are common not only in clinical settings but also in the general population, and are a major health issue in practically all areas of medicine [1,2]. Although there is no fixed list of functional somatic syndromes and every medical specialty has at least one such syndrome [1,3], they are known for considerable overlap as well as for comorbid symptoms and risk factors in common [4]. Nevertheless, little is known about the aetiology of the syndromes and underlying mechanisms of the overlap.

In our previous study [5], we proposed a model that explains genetic and environmental structure of these syndromes and estimated the relative importance of factors in common to the syndromes. However, a limitation of the previous study was that the definitions of these syndromes were based on existing classification criteria (e.g. 1994 criteria for chronic fatigue syndrome [6]), which has not been empirically confirmed yet and are possibly inclined to identify individuals with multiple symptoms.

Thus, in the present study we analyzed nine functional somatic symptoms instead of syndromes with an attempt to cover a wide variety of medical specialties: abdominal discomfort and gastroesophageal reflux from gastroenterology; general muscular pain from rheumatology; chronic fatigue from (possible) infectious diseases; dizziness from cardiology including autonomic dysfunction; breathlessness at rest from respiratory medicine; back pain from orthopaedics; and recurrent headache from neurology. To cluster these symptoms without assuming the current classification criteria for the syndromes to be valid, we employed latent class analysis, a method to test empirically for the existence of discrete groups who endorse similar patterns of symptoms $[7,8]$. We hypothesized that the symptoms could be partly lumped and partly splitted, which is in line with the main finding of our previous study [5]. Furthermore, as was the case in our previous study, we used a population-based sample of Swedish twins from the Swedish Twin Registry $[9,10]$. This genetically informative sample allowed us to examine whether genetic factors are important for a particular discrete group, if any.

\section{METHODS}

\section{Subjects}

Subjects were participants in the Swedish Twin Registry, which comprises all twin births in Sweden from 1886 to 2000, with data on place of birth, current vital status, and address of more than 160,000 individuals $[9,10]$. All living, contactable, and consenting twins born in Sweden between January 1935 and December 1958 were interviewed. All the participants provided verbal informed consent during this telephone interview, which was later confirmed by postcard. Zygosity was based on responses to questions regarding physical similarity in childhood. This method has been validated repeatedly as having $99 \%$ or higher accuracy by using DNA markers [9]. This study was approved by the ethical committee of The Karolinska Institute. 


\section{Interview procedures}

We screened all living, contactable, interviewable, and consenting twins born in Sweden for a wide range of health conditions including functional somatic symptoms and syndromes. The screening was conducted over a four year period from March 1998 to December 2002. Due to the confounding influence of ageing, questions about chronic fatigue were only asked of twins aged 41-64 years (i.e. born between 1 January 1935 and 31 December 1958). Because of this, the analysis in the present study was restricted to subjects aged 41-64. All interviews were conducted by trained personnel with adequate medical background using a computer based data collection system.

\section{Assessments of functional somatic syndromes or symptoms}

Screening of chronic fatigue was based on our criteria that emulated closely the 1994 criteria for chronic fatigue syndrome [6] without clinical examinations. We defined fatigue as the presence of self-reported abnormal, persistent tiredness in the last six months in the absence of an exclusionary condition. Exclusionary conditions were determined from multiple sources (e.g. Swedish national registers and medical records), as described elsewhere [11]. Interviewees were then asked whether they experienced impairment that affected at least $25 \%$ of their work. Finally, interviewees were asked about eight ancillary symptoms during the period of abnormal tiredness (substantial impairment in short-term memory or concentration; sore throat; tender lymph nodes; muscle pain; multi-joint pain without swelling or redness; headaches of a new type, pattern, or severity; unrefreshing sleep; and post-exertional malaise lasting more than 24 hours). The presence of four or more of these symptoms are an integral part of the definition of chronic fatigue syndrome [6]. Idiopathic chronic fatigue, which is also included in the 1994 criteria [6], was defined as having abnormal fatigue lasting at least six months and working impairment without reference to the number of ancillary symptoms. The definitions of chronic fatigue syndrome and idiopathic chronic fatigue correspond to $\mathrm{CF}-\mathrm{C}$ and $\mathrm{CF}-\mathrm{B}$ in our previous report [11].

General muscular pain was screened using our previously reported algorithm [12,13] based on the classification criteria for fibromyalgia proposed by the American College of Rheumatology [14] without clinical examinations such as tender point assessment. Interviewees were asked about widespread pain during the last three months in both the upper and lower body and in both the right and left sides of the body. Those who further endorsed pain on body axis (assessed as back pain in the last 12 months) in addition to the previous questions were defined as chronic widespread pain cases. Detailed information about the criteria for chronic widespread pain in this study was reported elsewhere [12]. In addition, we asked about the presence or absence of back pain in the past 12 months.

Gastrointestinal symptoms were assessed in the interview as described in our previous studies $[15,16]$ although instead of lifetime prevalence, symptoms during the past one year were used in the present study. We first asked whether interviewees had recurrent abdominal discomfort lasting at least seven days per month (approximately $25 \%$ of the time) during the past year. Interviewees were then asked about the presence or absence of recurrent pain in the upper abdomen, in the lower abdomen, or in another part of abdomen. Those who endorsed this question were further asked whether the pain was more prominent when feces became looser and defecation more frequent, and whether the pain stopped or alleviated just after defecation. Finally, individuals who reported a history of Crohn's disease, ulcerative colitis, stomach ulcers, or intestinal ulcers were excluded. Those who endorsed all the questions in the absence of an exclusionary condition were defined as having irritable bowel syndrome. This definition may be considered as an adapted version of Rome II criteria. In addition, gastroesophageal reflux was screened by using our algorithm $[17,18]$, defined as the occurrence, at least once a week, of either retrosternal pain or burning with antacid 
relief; or regurgitation of bitter fluid. Cases with gastroesophageal symptoms due to known causes (e.g. Crohn's disease) were excluded.

A lifetime history of recurrent headache not associated with infection, fever or hangover was assessed by the algorithm used in our previous reports [5,13,19], which is in line with the International Headache Society criteria.

The presence of urinary tract problems, dizziness and breathlessness at rest was based on a question about lifetime occurrence of each symptom.

\section{Assessment of Comorbid Psychiatric Disorders}

Major depression and generalized anxiety disorder were assessed by using the Computerized Composite International Diagnostic Interview-Short Form (CIDI-SF) adapted from its original design for 12-month prevalence to assess lifetime prevalence [20]. Details on these criteria were described elsewhere [21,22].

\section{Statistical Analyses}

Latent class analysis is a form of categorical data analysis hypothesizing that it is possible to account for the observed symptom endorsement profiles of respondents in terms of some small number of mutually exclusive respondent classes. We used the following nine somatic symptoms in this analysis: three cardinal symptoms of the three functional somatic syndromes, that is, abnormal tiredness lasting at least six months (without any exclusionary condition), widespread pain lasting at least three months, and abdominal discomfort lasting at least seven days per month in the past year; and six bodily symptoms as lifetime occurrence, that is, prolonged joint pain, back pain, gastroesophageal reflux, recurrent headache, recurrent urinary problem, and dizziness.

Latent class analysis was performed with the program LatentGOLD version 4.5 (Vermunt and Magidson 2005, Statistical Innovations Inc. Belmont, MA, USA). To determine the optimal number of latent classes, models estimating 1-class through 10-class solutions were compared. To select the best fitting model, we compared the change in the Bayesian Information Criterion (BIC), which is a goodness-of-fit index that considers the rule of parsimony. Once the number of classes was determined, the individuals were assigned to one of the latent classes based on their response profiles. Analysis of the response profiles provides important information about the nature of each class. Further understanding can be obtained by analysing additional data not entered into the model (e.g. age, gender, and the prevalence of relevant syndromes or illnesses).

Because our sample population was twins, we further performed structural equation modeling by which individual differences in the probability of being assigned to each latent class can be decomposed into three sources of variation: additive genetic, shared environmental, and nonshared environmental variance. Additive genetic effects are indicated when monozygotic twins are more similar than dizygotic twins. Shared (family) environmental effects (environmental effects that make family members similar) contribute equally to the similarity of monozygotic and dizygotic twins. Nonshared environmental effects do not contribute to twin similarity, and their importance is reflected in the extent to which monozygotic correlations are less than one. Maximum likelihood model fitting was applied to raw data of pair respondents (i.e. both of the twin pair responded) by using the Mx program [23]. First, we estimated parameters for men and women separately, and then tested if the parameters could be equated. Second, we compared the full model (composed of additive genetic, shared environmental, and nonshared environmental effects) with its nested models by dropping one of the parameters. A significant change in goodness-of-fit indicates that the nested model is worse than the full model. 


\section{RESULTS}

Of 41355 eligible individuals aged 41-64 years, 28531 individuals provided the complete sets of responses for this study (69.0\%). Data were obtained from both members of 6560 monozygotic or same-sexed dizygotic twin pairs. Table 1 shows the characteristics of the respondents by gender. The gender distribution had a slight female predominance, but the mean age was almost identical for both genders.

Table 2 shows gender-specific prevalence data for three major functional somatic syndromes and two comorbid psychiatric disorders. The prevalence for all the three syndromes was smaller compared to the literature [26-28], presumably due to different definitions.

A series of latent class analysis revealed that, based on BIC, the optimal number of latent classes is five. Table 3 shows the symptom profiles for the 5-class solution, by which a total of 28531 respondents were assigned to one of the five classes. The mean age was similar across the classes, whereas male-female ratios differed depending on the class. The majority $(66 \%)$ of the total were classified into Class 1 , in which almost none of them had any symptoms except back pain. In Class 2 through Class 5, women were more prominent than men, particularly in Class 5. Some individuals in Class 2 tended to feel abnormally tired but had neither widespread pain nor abdominal discomfort. On the other hand, those in Class 2 showed relatively high prevalence of dizziness, a symptom of orthostatic intolerance. All individuals assigned to Class 3 endorsed recurrent abdominal discomfort, and many of them had gastroesophageal reflux as well. In contrast, all individuals in Class 4 endorsed widespread pain lasting at least three months, and most had back pain as well. Finally, those assigned to Class 5 manifested the highest proportions for almost all the symptoms analyzed.

The characterization of each class can further be explored by comparing the prevalences for comorbid illnesses or conditions shown in Table 4. As can be expected, individuals in Class 5 showed the highest prevalences for most of the syndromes or disorders. Only chronic widespread pain showed its highest prevalence in Class 4 and irritable bowel syndrome in Class 3. Although Class 2 included $10.6 \%$ of individuals having idiopathic chronic fatigue (without regard to the ancillary symptoms), the prevalence of chronic fatigue syndrome (with four or more ancillary symptoms) was only $2.6 \%$. The two psychiatric disorders differed from class to class, but sparsely distributed in general.

Table 5 summarizes the results of model fitting for the probability of each class membership. Because testing gender differences in parameter estimates turned out to be significant except Class 3 and dropping shared environmental effects resulted in no significant change in goodness-of-fit for all the classes, we show the proportion of variance due to additive genetic and nonshared environmental effects stratified by gender. As expected, the relative importance of genetic effects (i.e. heritability) was small but significant across the classes. Albeit statistically significant, gender differences in heritability estimate were not prominent in any class. Furthermore, the relative importance of nonshared environmental effects was pronounced in all the classes.

\section{DISCUSSION}

The present study clearly demonstrates the heterogeneity of three major functional somatic syndromes and thus the necessity of re-defining each "single" syndrome based on empirical evidence. This study also provides information about the extent to which the possible redefined syndromes are influenced by genetic and environmental factors in their aetiology. 
The most remarkable finding in this study is that individuals with functional somatic symptoms can be categorized into a number of statistically distinct classes depending on which and how many symptoms they have. First, individuals assigned to Class 5 by our latent class analysis ( $2.8 \%$ of the total sample) reported that they had a multitude of bodily symptoms (Table 3). Although we did not screen somatoform disorder in this study, it is likely that those individuals would be considered so-called "somatizers". The high prevalences of chronic fatigue, chronic widespread pain, irritable bowel syndrome as well as major depression and generalized anxiety disorder in Class 5 (Table 4) suggest that people who would fall in our Class 5 are the source of high comorbidity among these syndromes or disorders. Second, individuals in Class 4 (3.4\%) tended to report pain-related symptoms in particular (Table 3), and accordingly, that class experienced the highest prevalence of chronic widespread pain (Table 4). Third, individuals assigned to Class 3 (3.9\%) showed higher proportions of gastrointestinal symptoms (i.e. recurrent abdominal discomfort and gastroesophageal reflux) in Table 3 and thus irritable bowel syndrome was highest in Table 4. Those with irritable bowel syndrome but without chronic widespread pain could be distinguished from those with chronic widespread pain but without irritable bowel syndrome. Furthermore, we found that individuals in Class 2 (24.3\%) tended to have relatively high prevalence of idiopathic chronic fatigue (10.6\%), whereas almost none of them met either chronic widespread pain or irritable bowel syndrome criteria. Instead, those in Class 2 showed a higher proportion of dizziness, a symptom of orthostatic intolerance that is possibly associated with autonomic dysfunction in chronic fatigue cases [27]. Taken together, overlaps among the three functional somatic syndromes were not substantial except in Class 5.

It is of particular interest that we observed the two clustering patterns of somatic symptoms: single-symptom/syndrome classes (Classes 2, 3 and 4) and a multiple- symptom class (Class 5). Distinction among the single-symptom/syndrome classes was relatively clear, especially between Class 3 (gastrointestinal problem-prone class) and Class 4 (chronic pain-prone class). Even though abnormal tiredness in Class 2 was not salient relative to other classes, its high prevalence of idiopathic chronic fatigue suggests that we could capture a sort of pure chronic fatigue cases without so-called ancillary symptoms. In other words, patients with multiple comoribidity (or functional somatic syndromes) were well represented on Class 5 (somatisation-prone class). We emphasize here that this classification was not influenced by any a priori assumptions or beliefs, but was derived solely from a statistical technique. These findings tempt us to say that the appropriate question about functional somatic syndromes is not "one or many" [1] but "single or multiple". Importantly, we found that the prevalence of psychiatric comorbidity (major depression and generalized anxiety disorder) dispersed across the classes. This can be considered an empirical support for the notion [28] that even single syndromes probably entail psychosocial and cultural factors as well as biological factors in their aetiology. In other words, it is more helpful to distinguish between uncomplicated syndromes and complicated syndromes [2], rather than simplified dualism such as "organic or psychogenic".

Another important implication of our findings comes from a comparison of fatigue-related syndromes across the classes: It seems that there is a fundamental flaw in the 1994 criteria for chronic fatigue syndrome [6]. Although Classes 2 and 3 showed relatively highe prevalence for idiopathic chronic fatigue, approximately three quarters of the cases did not meet the criteria for chronic fatigue syndrome (based on self-report). Such a substantial reduction was not observed in Classes 4 and 5, which showed higher prevalence for idiopathic chronic fatigue. Thus, it is likely that the "four or more" criterion of the 1994 criteria contributes to omitting cases with chronic fatigue as a single syndrome (as in Class 2) and instead introduces cases with complicated symptoms (as in Class 5). This finding is consistent with our previous report using the same sample of twins [29], where we found 
little support for the "four or more" criterion based on different approaches and instead found that having muscle and multi-joint pain was the best predictor for being diagnosed with chronic fatigue syndrome. We therefore suggest again that the ancillary symptom criterion should be dropped entirely, and that more attention should be paid to individuals with idiopathic (i.e. pure) chronic fatigue.

As our subjects were twins, we could go further and examine the relative importance of genetic and environmental influences on the probability of class membership. Albeit small, we detected genetic influences on the probability of class membership for all the five classes in both genders. Several studies have estimated the heritability of functional somatic syndromes, including our own $[12,16,26,30]$. However, these previous studies used existing criteria for the syndromes making it difficult to compare directly with genetic influences on the putatively pure syndromes found in the present study. In addition, a few twin studies found significant genetic and no family environmental factors for somatic distress in both men and women $[31,32]$, but the estimates varied. Further work is thereby needed to evaluate the extent to which genetic factors influence liability to each of the single syndromes and the condition with multiple somatic symptoms.

The present study has notable strengths such as using a genetically informative sample of twins who can be considered a representative of Swedish population. In addition, three major functional somatic syndromes and relevant health conditions were assessed by using a structured interview in a blinded manner, which allows us to minimize recall and interviewer biases. Furthermore, the final model with the optimal number of latent class was selected without a priori assumptions or biases due to investigators' medical specialty [1,2]. Nevertheless, some limitations should also be noted. First, our assessment was based on self-reports via telephone interviews, without medical examinations by a physician. Thus, the results may not be directly comparable to those using clinical samples. Second, the data were obtained on only one occasion, although some of the illnesses were diagnosed as lifetime occurrence. Thus, temporal order is unclear. Third, the latent class analysis solution is dependent on the input dataset. Thus, a different set of data may yield a different latent class analysis solution. Finally, the assumption of local independence for latent class analysis may be violated in part because it is unlikely that all the indicator variables (i.e. symptoms) in the model are independent of one another; however, this possible violation of local independence should not affect the determination of the optimal number of latent classes.

In conclusion, the research on functional somatic syndromes and symptoms needs to shift from the stage of asking whether functional somatic syndromes are "one or many" [1,33] or sticking to the dualistic view of "organic versus psychogenic". The findings in the present study suggest that the researchers need to re-define these syndromes by distinguishing uncomplicated, single syndromes from complicated, multiple ones. Such integrated approaches across medical specialties will promote better understandings of the aetiology and then lead to improved management.

\section{Acknowledgments}

This work was supported by NS-041483 (NLP), CA-085739 (PFS), and AI-056014 (PFS). The Swedish Twin Registry is supported by grants from the Swedish Department of Higher Education, the Swedish Scientific Council, and an unrestricted grant from AstraZeneca.

\section{REFERENCES}

1. Wessely S, Nimnuan C, Sharpe M. Functional somatic syndromes: one or many? Lancet 1999;354(9182):936-939. [PubMed: 10489969] 
2. Henningsen P, Zipfel S, Herzog W. Management of functional somatic syndromes. Lancet 2007;369(9565):946-955. [PubMed: 17368156]

3. Kanaan RAA, Lepine JP, Wessely SC. The association or otherwise of the functional somatic syndromes. Psychosom Med 2007;69(9):855-859. [PubMed: 18040094]

4. Aaron LA, Herrell R, Ashton S, Belcourt M, Schmaling K, Goldberg J, et al. Comorbid clinical conditions in chronic fatigue: a co-twin control study. J Gen Intern Med 2001;16(1):24-31. [PubMed: 11251747]

5. Kato K, Sullivan PF, Evengård B, Pedersen NL. A population-based twin study of functional somatic syndromes. Psychol Med 2009;39(3):497-505. [PubMed: 18578896]

6. Fukuda K, Straus SE, Hickie I, Sharpe MC, Dobbins JG, Komaroff A. International Chronic Fatigue Syndrome Study Group. The chronic fatigue syndrome: a comprehensive approach to its definition and study. Ann Intern Med 1994;121(12):953-959. [PubMed: 7978722]

7. Sullivan PF, Smith W, Buchwald D. Latent class analysis of symptoms associated with chronic fatigue syndrome and fibromyalgia. Psychol Med 2002;32(5):881-888. [PubMed: 12171382]

8. Sullivan PF, Kessler RC, Kendler KS. Latent class analysis of lifetime depressive symptoms in the national comorbidity survey. Am J Psychiatry 1998;155(10):1398-1406. [PubMed: 9766772]

9. Lichtenstein P, De Faire U, Floderus B, Svartengren M, Svedberg P, Pedersen NL. The Swedish Twin Registry: a unique resource for clinical, epidemiological and genetic studies. J Intern Med 2002;252(3):184-205. [PubMed: 12270000]

10. Lichtenstein P, Sullivan PF, Cnattingius S, Gatz M, Johansson S, Carlstrom E, et al. The Swedish Twin Registry in the third millennium: an update. Twin Res Hum Genet 2006;9(6):875-882. [PubMed: 17254424]

11. Evengård B, Jacks A, Pedersen NL, Sullivan PF. The epidemiology of chronic fatigue in the Swedish Twin Registry. Psychol Med 2005;35(9):1317-1326. [PubMed: 16168154]

12. Kato K, Sullivan PF, Evengård B, Pedersen NL. Importance of genetic influences on chronic widespread pain. Arthritis Rheum 2006;54(5):1682-1686. [PubMed: 16646040]

13. Kato K, Sullivan PF, Evengård B, Pedersen NL. Chronic widespread pain and its comorbidities: a population-based study. Arch Intern Med 2006;166(15):1649-1654. [PubMed: 16908799]

14. Wolfe, F.; Smythe, HA.; Yunus, MB.; Bennett, RM.; Bombardier, C.; Goldenberg, DL., et al. The American College of Rheumatology 1990 Criteria for the Classification of Fibromyalgia. Report of the Multicenter Criteria Committee; Arthritis Rheum. 1990. p. 160-172.

15. Svedberg P, Johansson S, Wallander MA, Hamelin B, Pedersen NL. Extra-intestinal manifestations associated with irritable bowel syndrome: a twin study. Aliment Pharmacol Ther 2002;16(5):975983. [PubMed: 11966507]

16. Wojczynski MK, North KE, Pedersen NL, Sullivan PF. Irritable bowel syndrome: a co-twin control analysis. Am J Gastroenterol 2007;102(10):2220-2229. [PubMed: 17897337]

17. Cameron AJ, Lagergren J, Henriksson C, Nyren O, Locke GR 3rd, Pedersen NL. Gastroesophageal reflux disease in monozygotic and dizygotic twins. Gastroenterology 2002;122(1):55-59. [PubMed: 11781280]

18. Zheng Z, Nordenstedt H, Pedersen NL, Lagergren J, Ye W. Lifestyle factors and risk for symptomatic gastroesophageal reflux in monozygotic twins. Gastroenterology 2007;132(1):87-95. [PubMed: 17241862]

19. Svensson DA, Larsson B, Waldenlind E, Pedersen NL. Genetic and environmental influences on expression of recurrent headache as a function of the reporting age in twins. Twin Res 2002;5(4): 277-286. [PubMed: 12217234]

20. Kessler RC, Andrews G, Mroczek CK, Ustun B, Wittchen H-U. The World Health Organization Composite International Diagnostic Interview Short Form (CIDI-SF). Int J Methods Psychatric Res 1998;7:171-185.

21. Kendler KS, Gatz M, Gardner CO, Pedersen NL. A Swedish national twin study of lifetime major depression. Am J Psychiatry 2006;163(1):109-114. [PubMed: 16390897]

22. Mackintosh MA, Gatz M, Wetherell JL, Pedersen NL. A twin study of lifetime generalized anxiety disorder (GAD) in older adults: genetic and environmental influences shared by neuroticism and GAD. Twin Res Hum Genet 2006;9:30-37. [PubMed: 16611465] 
23. Neale, MC.; Boker, SM.; Xie, G.; Maes, HHM. Mx: Statistical Modeling. Richmond, VA: Virginia Commonwealth University Department of Psychiatry; 2004.

24. Watanabe N, Stewart R, Jenkins R, Bhugra DK, Furukawa TA. The epidemiology of chronic fatigue, physical illness, and symptoms of common mental disorders: a cross-sectional survey from the second British National Survey of Psychiatric Morbidity. J Psychosom Res 2008;64(4): 357-362. [PubMed: 18374734]

25. Aggarwal VR, McBeth J, Zakrzewska JM, Lunt M, Macfarlane GJ. The epidemiology of chronic syndromes that are frequently unexplained: do they have common associated factors? Int $\mathrm{J}$ Epidemiol 2006;35(2):468-476. [PubMed: 16303810]

26. Lembo AJ, Zaman M, Krueger RF, Tomenson BM, Creed FH. Psychiatric disorder, irritable bowel syndrome, and extra-intestinal symptoms in a population-based sample of twins. Am J Gastroenterol 2009;104(3):686-694. [PubMed: 19223882]

27. Schondorf R, Freeman R. The importance of orthostatic intolerance in the chronic fatigue syndrome. Am J Med Sci 1999;317(2):117-123. [PubMed: 10037115]

28. Henningsen P, Herzog W. Irritable bowel syndrome and somatoform disorders. J Psychosom Res 2008;64(6):625-629. [PubMed: 18501264]

29. Sullivan PF, Pedersen NL, Jacks A, Evengård B. Chronic fatigue in a population sample: definitions and heterogeneity. Psychol Med 2005;35(9):1337-1348. [PubMed: 16168156]

30. Sullivan PF, Evengård B, Jacks A, Pedersen NL. Twin analyses of chronic fatigue in a Swedish national sample. Psychol Med 2005;35(9):1327-1336. [PubMed: 16168155]

31. Gillespie NA, Zhu G, Heath AC, Hickie IB, Martin NG. The genetic aetiology of somatic distress. Psychol Med 2000;30(5):1051-1061. [PubMed: 12027042]

32. Kendler KS, Walters EE, Truett KR, Heath AC, Neale MC, Martin NG, et al. A twin-family study of self-report symptoms of panic-phobia and somatization. Behav Genet 1995;25(6):499-515. [PubMed: 8540889]

33. Wessely S, White PD. There is only one functional somatic syndrome. Br J Psychiatry 2004;185:95-96. [PubMed: 15286058] 
Table 1

Descriptive statistics of the respondents

\begin{tabular}{lccc}
\hline & Men & Women & Total \\
\hline Number of respondents $(\%)$ & 13685 & 14846 & 28531 \\
& $(48.0 \%)$ & $(52.0 \%)$ & \\
Number of intact twin pairs & & & 6560 \\
Monozygotic twins & 1249 & 1530 & 2779 \\
Same-sexed dizygotic twins & 1762 & 2019 & 3781 \\
Mean age (years \pm SD) & $53.7 \pm 5.7$ & $53.7 \pm 5.7$ & \\
\hline
\end{tabular}

J Psychosom Res. Author manuscript; available in PMC 2011 May 1. 


\section{Table 2}

Gender-specific prevalence of functional somatic syndromes and their comorbid psychiatric disorders

\begin{tabular}{lccc}
\hline & Men & Women & Total \\
\hline Prevalence of functional somatic syndromes $(\%)$ & & \\
Chronic fatigue syndrome * & 0.9 & 3.3 & 2.1 \\
Chronic widespread pain & 1.9 & 6.1 & 4.1 \\
$\begin{array}{l}\text { Irritable bowel syndrome } \\
\text { Prevalence of comorbid psychiatric disorders }(\%)\end{array}$ & 1.4 & 1.0 \\
$\begin{array}{l}\text { Major depression (lifetime) } \\
\text { Generalized anxiety disorder } \\
\text { (lifetime) }\end{array}$ & 15.6 & 27.9 & 22.0 \\
\hline
\end{tabular}

* Clinical examinations required for the diagnosis of chronic fatigue were not performed. 


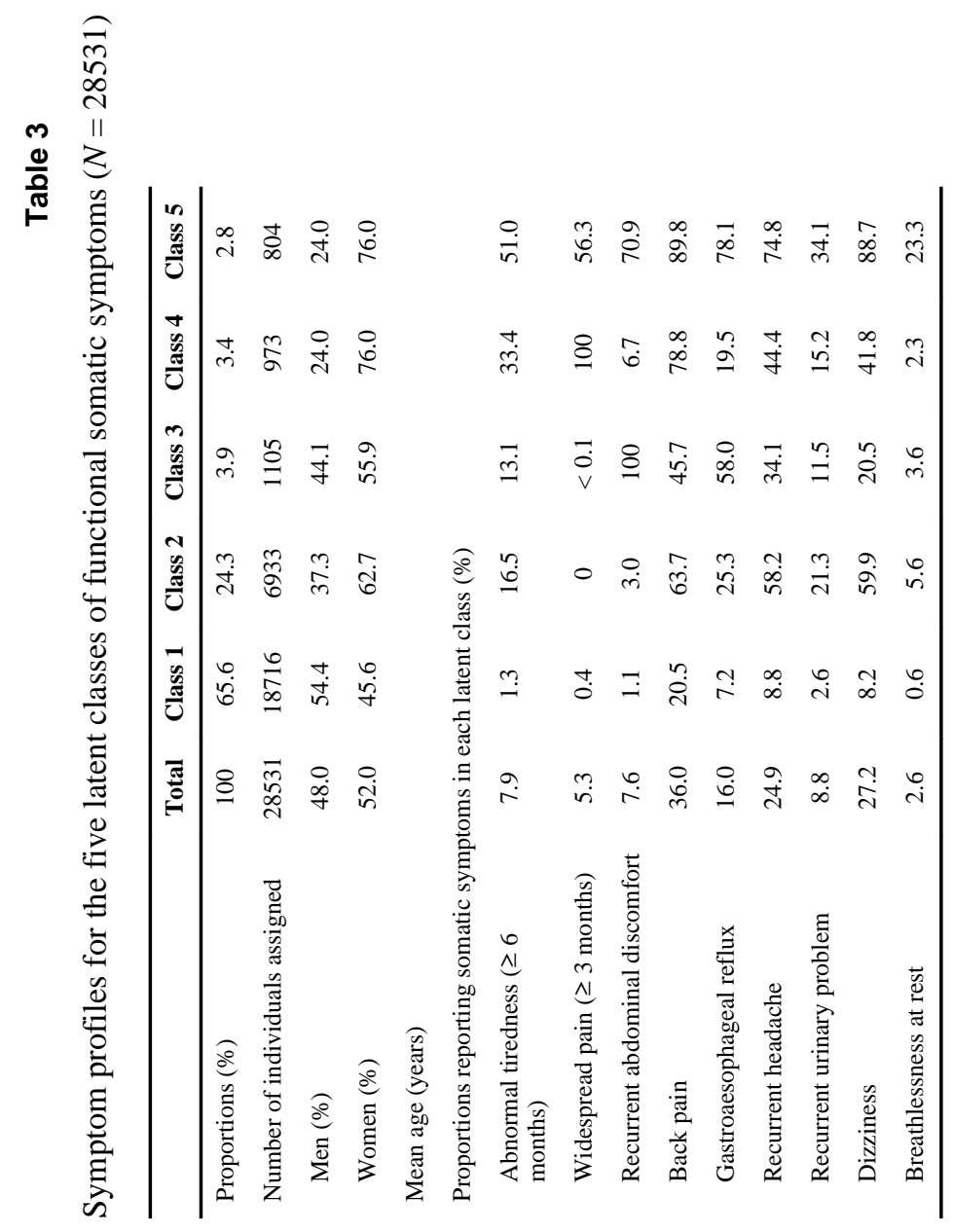


Table 5

Gender-specific estimates of proportions of variances (with 95\% confidence intervals) and a goodness-of-fit index for the probability of class membership

\begin{tabular}{|c|c|c|c|c|}
\hline & & \multicolumn{2}{|c|}{$\begin{array}{l}\text { Proportions of variances } \\
\text { (95\% confidence interval) }\end{array}$} & \multirow{2}{*}{$\begin{array}{c}-2 L L \\
(d f=13114)^{*}\end{array}$} \\
\hline & & $\begin{array}{l}\text { Additive } \\
\text { genetic }\end{array}$ & $\begin{array}{c}\text { Nonshared } \\
\text { environmental }\end{array}$ & \\
\hline \multirow[t]{2}{*}{ Class 1} & Men & $\begin{array}{c}0.27 \\
(0.23-0.32)\end{array}$ & $\begin{array}{c}0.73 \\
(0.68-0.77)\end{array}$ & 7873.01 \\
\hline & Women & $\begin{array}{c}0.29 \\
(0.25-0.33)\end{array}$ & $\begin{array}{c}0.69 \\
(0.67-0.75)\end{array}$ & \\
\hline \multirow[t]{2}{*}{ Class 2} & Men & $\begin{array}{c}0.17 \\
(0.12-0.21)\end{array}$ & $\begin{array}{c}0.83 \\
(0.79-0.88)\end{array}$ & 494.26 \\
\hline & Women & $\begin{array}{c}0.14 \\
(0.10-0.18)\end{array}$ & $\begin{array}{c}0.86 \\
(0.82-0.90)\end{array}$ & \\
\hline Class $3^{* *}$ & & $\begin{array}{c}0.07 \\
(0.04-0.10)\end{array}$ & $\begin{array}{c}0.93 \\
(0.90-0.96)\end{array}$ & -14373.97 \\
\hline \multirow[t]{2}{*}{ Class 4} & Men & $\begin{array}{c}0.09 \\
(0.05-0.14)\end{array}$ & $\begin{array}{c}0.91 \\
(0.89-0.94)\end{array}$ & -15630.42 \\
\hline & Women & $\begin{array}{c}0.13 \\
(0.08-0.17)\end{array}$ & $\begin{array}{c}0.87 \\
(0.83-0.92)\end{array}$ & \\
\hline \multirow[t]{2}{*}{ Class 5} & Men & $\begin{array}{c}0.16 \\
(0.11-0.21)\end{array}$ & $\begin{array}{c}0.84 \\
(0.79-0.89)\end{array}$ & -18266.76 \\
\hline & Women & $\begin{array}{c}0.21 \\
(0.16-0.25)\end{array}$ & $\begin{array}{c}0.79 \\
(0.75-0.84)\end{array}$ & \\
\hline
\end{tabular}

